\title{
Metastatic Intracavitary Cardiac Aortic Body Tumor in a Dog
}

\author{
Kyoung-Oh CHO, Nam-Yong PARK ${ }^{1)}$, In-Chul PARK ${ }^{3)}$, Byung-Kyu KANG ${ }^{2)}$ and Misao ONUMA \\ Laboratory of Infectious Diseases, Department of Disease Control, Graduate School of Veterinary Medicine, Hokkaido University, \\ Sapporo 060-0818, Japan, ${ }^{1)}$ Departments of Veterinary Pathology, ${ }^{3)}$ Veterinary Theriogenology, and ${ }^{2)}$ Veterinary Teaching Hospital, \\ College of Veterinary Medicine, Chonnam National University, Kwang-Ju, Korea \\ (Received 17 March 1998/Accepted 25 June 1998)
}

ABSTRACT. A mobile right-ventricular mass dynamically occluding the right ostium atrioventriculare in the systolic phase was detected in a 3-year-old male Tosa dog by echocardiography. At necropsy, multiple tumor masses of various sizes were observed in the heart base, right ventricular lumen, myocardium, lung and liver. Dysplasia of tricuspid valve characterized by irregular shape of leaflets, upward malposition of large papillary muscles, and shortened and stout chordae tendineae was also detected. Histopathologically, the tumor cells, arranged in sheets or nests, were polyhedral with lightly eosinophilic and finely granular cytoplasm, and contained a hyperchromatic round or oval nucleus. By Grimelius' silver stain, tumor cells had cytoplasmic positive granules. Ultrastructurally, tumor cells contained characteristic small membrane-limited granules. This is the first report of metastatic intracavitary cardiac aortic body tumor in a dog. — KEY WORDS: aortic body tumor, intracavitary metastase, tricuspid valve dysplasia.

Although chemoreceptor tissue is found at several sites in the body of animals [5], tumors arising only in the aortic and carotid bodies have been reported [1,2]. The tumor arising from the aortic bulb is called as aortic body tumor and is encountered more frequently than carotid body tumor in animals $[1,2]$. Aortic body tumor in animals is nonfunctional and space-occupying lesions, and may cause a variety of cardiac functional disturbances [1, 2].

Dysplasia of the tricuspid valve is a cardiac malformation and is rarely observed in dogs $[6,8]$. It can induce tricuspid regurgitation, with a subsequent increase in volume and pressure in the right atrium, leading to cardiac syncope, peripheral circulatory failure and congestive heart failure $[6,8]$.

Here we report the intracavitary cardiac metastasis of aortic body tumor, and concurrent dysplasia of tricuspid valve in a 3-year-old male Tosa dog.

A 3-year-old male Tosa dog was presented to the Chonnam National University Veterinary Teaching Hospital, Korea. Clinical examination revealed hypothermia $\left(30^{\circ} \mathrm{C}\right)$, severe dyspnea, leukocytosis, emaciation and dehydration. By echocardiography, right atrium, right ventricle and pulmonary artery were found to be enlarged. In addition, a mobile mass measuring $2.2 \times 2.4 \mathrm{~cm}$ in diameter was detected in the right ventricle. The mass was free from the ventricular endocardium and occluded the right ostium atrioventriculare in the systolic phase. Slight regurgitation to right atrium was observed in the systolic phase. Splenomegaly and hepatomegaly were also detected. Radiographs exhibited variable sized abnormal masses throughout the lung. The dog had been dead and necropsied about $20 \mathrm{hr}$ after death.

At necropsy, cloudy, amber-colored fluid was observed in the thorax. A tumor mass measuring $3.5 \times 3.1 \mathrm{~cm}$ in diameter was found between the ascending aorta and pulmonary artery (Fig. 1). This tumor mass was coarsely rough on the surface, encapsulated and irregularly lobulated with yellow-brown central necrotic and thin peripheral white areas on the cut surface.

The right ventricular chamber was markedly dilated. In the lumen, there was a tumor mass measuring $3.3 \times 2.6 \mathrm{~cm}$ with yellow-brown central necrotic and thin peripheral white areas on the cut surface (Fig. 2). It was free from the ventricular wall and septum. Small white round tumor masses $(0.3 \mathrm{~cm}$ in diameter) were scattered in the right and left ventricular walls and ventricular septum.

Leaflets of the tricuspid valve were nodular and mildly thickened (Fig. 3). Elongation of the septal leaflet (which was adhered partly to the septum) and shortening of the lateral leaflet were observed. Chordae tendineae was short and stout. Papillary muscles were large and located upward to the leaflets. The right atrium was greatly dilated and

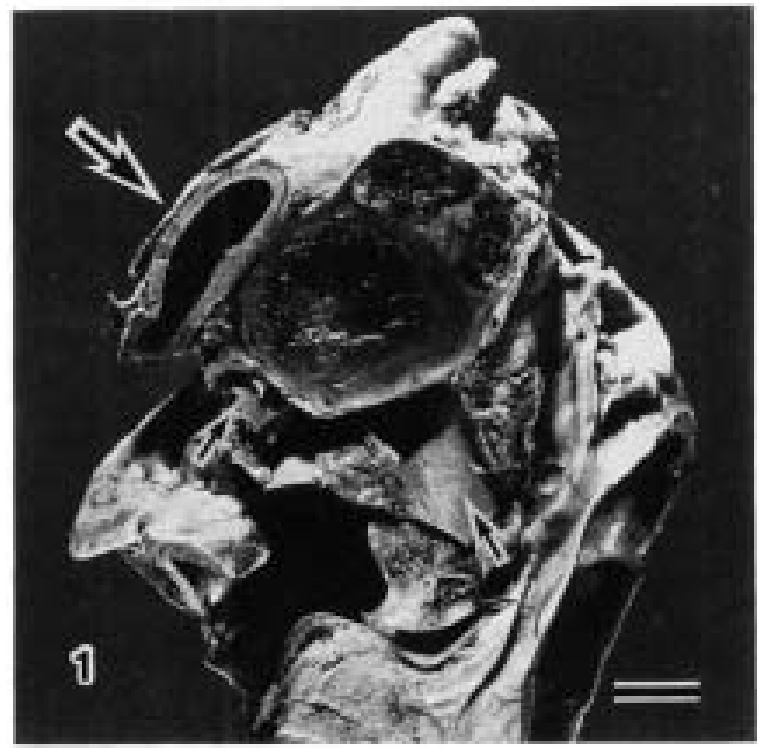

Fig. 1. Malignant aortic body tumor located between the ascending aorta (large arrow) and pulmonary artery (small arrows). Bar $=1 \mathrm{~cm}$. 


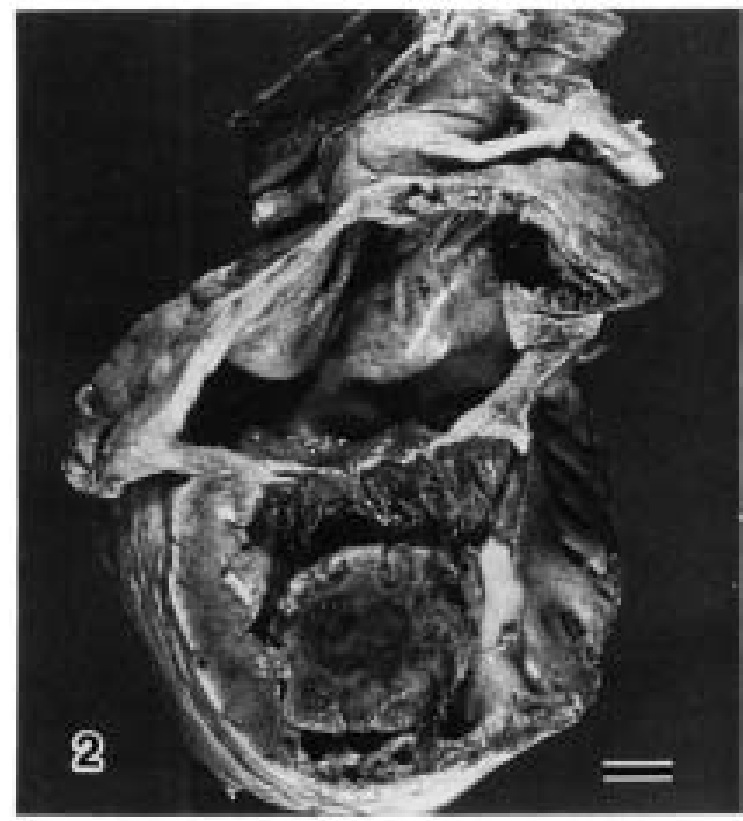

Fig. 2. Metastatic tumor mass free in the right ventricle. Bar $=1 \mathrm{~cm}$.

endocardium was white and thickened (Fig. 3)

Many white nodules up to $3 \mathrm{~cm}$ in diameter were scattered throughout the lung and liver.

Tissues were fixed in 10\% neutral buffered formalin and routinely processed for paraffin sections. The sections were stained with hematoxylin and eosin. Selected sections were stained with Grimelius' silver stain, periodic acid-Schiff (PAS), elastica van Gieson and Masson's trichrome. A part of the formalin-fixed tumor mass was rinsed in $0.1 \mathrm{M}$ phosphate buffer (PB), postfixed in $1 \%$ osmium tetraoxide, and embedded in Quetol (Epon 812) resin. Ultrathin sections were double-stained with uranyl acetate and lead citrate and examined using a JEOL JEM-1210 electron microscope.

The histopathological findings of all tumor masses in the heart, lung, and liver were similar, reading to lesions of tumors in myocardium, lung, and liver were thought to be metastatic from heart base aortic body tumor. Tumor cells were arranged in sheets or nests that were separated by fine fibrovascular connective tissue (Fig. 4). The cytoplasm of the cell was polyhedral, lightly eosinophilic, finely granular, and occasionally vacuolated (Fig. 5). The cytoplasmic boundaries were usually distinct, but occasionally indistinct. Some of the tumor cells contained Grimelius' silver- positive granules in the cytoplasm (Fig. 6). Nuclei varied in size and shape, but most was round to oval and usually centrally located (Fig. 5). Chromatin was usually abundant but occasionally largely clumped. Occasionally, giant cells were observed among tumor cells (Fig. 5). These giant cells were pleomorphic and characterized by a large bizarre hyperchromatic nucleus and moderate to abundant, lightly eosinophilic fine cytoplasmic granules. Mitotic figures were

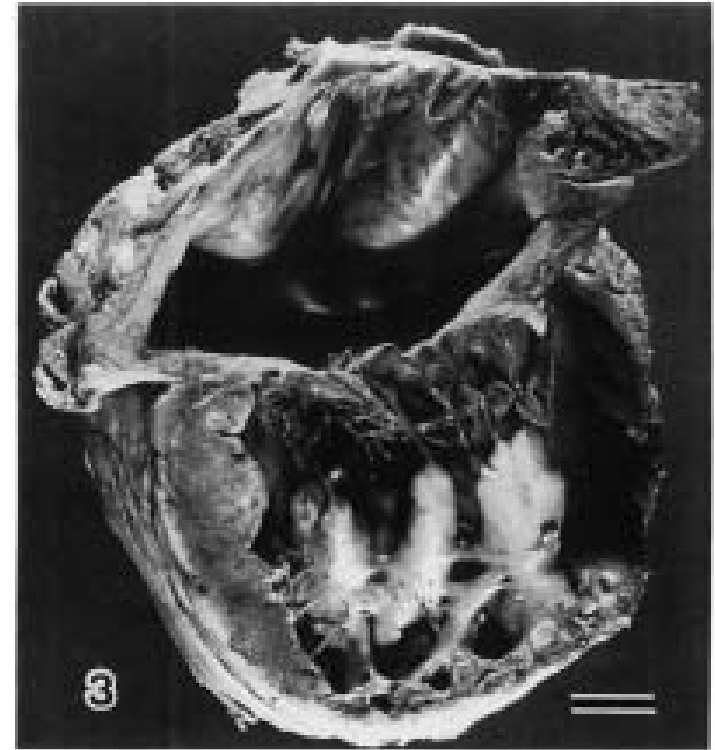

Fig. 3. Tricuspid valve dysplasia characterized by irregular leaflets in shape, upward malposition of large papillary muscles and short and shout chordae tendineae. Right ventricle and atrium are markedly dilated. Bar=1 $\mathrm{cm}$.

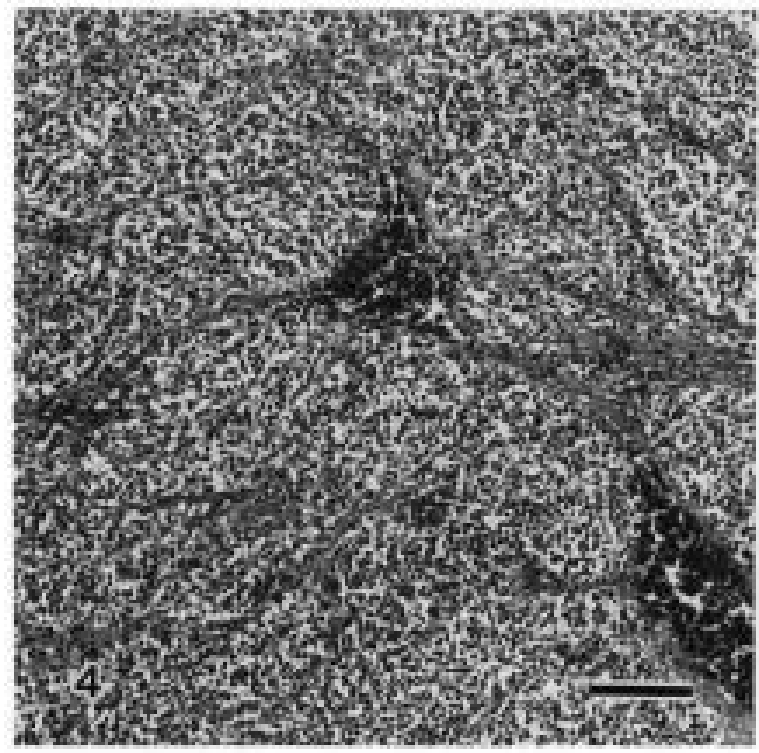

Fig. 4. Malignant aortic body tumor arranged in nests are separated by fine fibrovascular connective tissue. H\&E stain. $\mathrm{Bar}=100 \mu \mathrm{m}$.

infrequent. The large tumor masses in the heart base and the right ventricular lumen showed a wide necrotic area. Tumor cell emboli were observed in many blood vessels in the heart, lung and liver.

Ultrastructurally, few of the tumor cells contained small, dense membrane-bound granules, spherical mitochondria, and rough endoplasmic reticulum. They had large spherical 


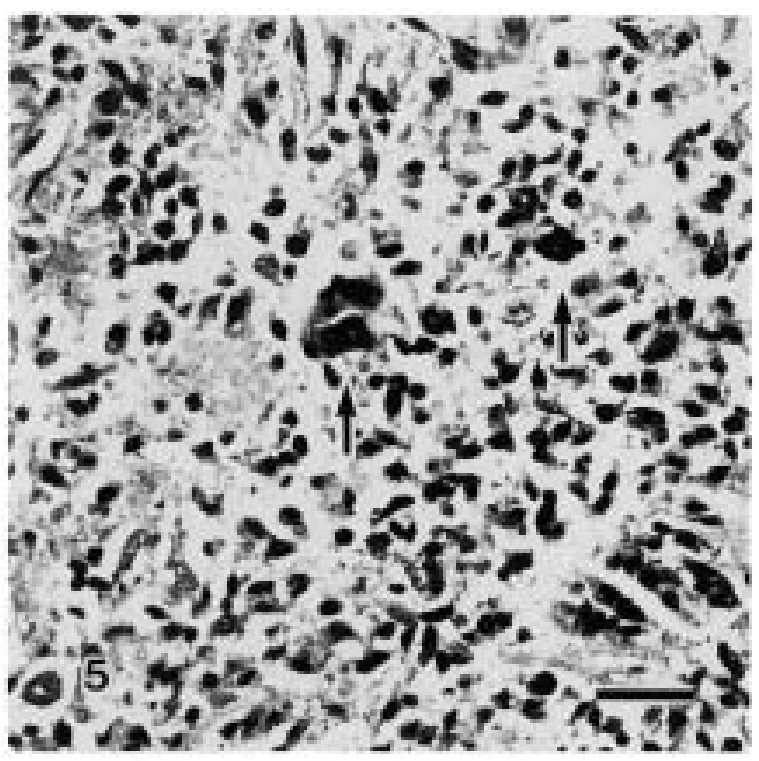

Fig. 5. The tumor cells are polyhedral with lightly eosinophilic cytoplasm and hyperchromatic round to oval nuclei. There are bizarre giant cells (arrows) among tumor cells. H\&E stain. Bar=30 $\mu \mathrm{m}$.

nuclei with clumped chromatin.

The tricuspid valve leaflets were moderately thickened and myxomatous in the spongiosa and, in addition, hypoelastic and irregular in the fibrosa. Papillary muscles in the right ventricle, and myocardium of the right atrium and ventricle showed mild hypertrophy characterized by increase of the length and diameter of the myofiber with irregular shaped large nuclei. Endocardium of the right atrium was extremely thickened by proliferation of fibrous tissues.

From the present results of histopathological and ultrastructural findings, the tumor was diagnosed as malignant aortic body tumor. Although myocardial metastases of a tumor from a primary lesions in other organs are common [1, 2], intracavitary metastases are uncommon both in animals and humans [3, 4, 7, 9]. Myocardial invasion of aortic body tumor is occasionally observed [1, 2], but intracavitary metastases of the tumor has never been reported in veterinary literature.

Generally, the right ventricle receives the systemic blood from the right atrium and pumps it to the lungs through the right outflow track. Therefore, if there would be a free large tumor mass in the right ventricle, it usually would obstruct the right ventricular outflow track [7, 9]. Interestingly, the present intracavitary tumor occluded the right ostium atrioventriculare in the systolic phase. In the present case, dysplasia of tricuspid valve might have induced regurgitation of ventricular blood into atrium during the systolic phase. This may cause occlusion of the ostium atrioventridulare by the tumor mass. However, it is still

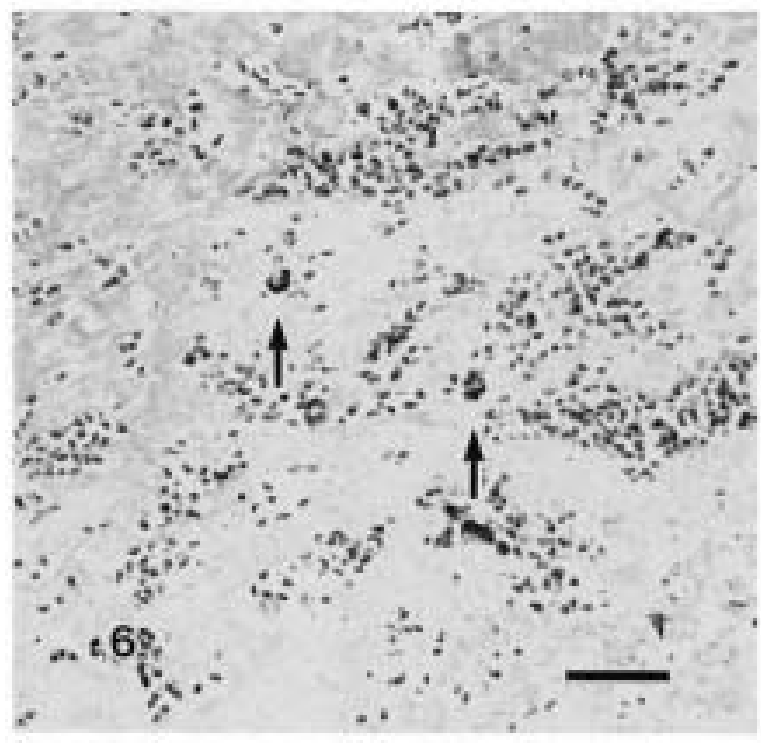

Fig. 6. Some of tumor cells (arrows) has Grimelius' silverpositive granules in the cytoplasm. Grimelius' silver stain. Bar $=50 \mu \mathrm{m}$.

possible that the obstruction of the ostium atrioventriculare by intracavitary tumor itself might cause mild regurgitation to right atrium in the present case .

ACKNOWLEDGMENT. The authors thank Dr. Y. Hanahusa for translation of abstract in Japanese and Dr. N.K. Rakha for revising the manuscript.

\section{REFERENCES}

1. Capen, C.C. 1990. pp. 553-639. In: Tumors in Domestic Animals, 3rd ed. (Moulton, J.E. ed.), University of California Press, Berkeley.

2. Capen, C.C. 1993. pp. 267-347. In: Pathology of Domestic Animals, 4th ed. (Jubb, K.V.F. ed.), Academic Press, San Diego.

3. Giannolo, B., Renzulli, A., Micheletti, E., Sante, P., Cerasuolo, F., Festa, M. and Cotrufo, M. 1987. Ital. J. Surg. Sci. 17: $117-122$.

4. Kuo, T-T., Yang, C-P., Lin, C-H. and Chang, C-H. 1987. Pediatr. Pathol. 7: 341-349.

5. LeCompte, P.M. 1951. pp. 1-40. In: Atlas of Tumor Pathology, Armed Forces Institute of Pathology, Washington, D.C.

6. Liu, S.K. and Tilley, L.P. 1976. J. Am. Vet. Med. Assoc. 15: 623-630.

7. Ports, T.A. Schiller, N.B. and Strunk, B.L. 1977. Circulation 56: 439-447.

8. Robinson, W.F. and Maxie, M.G. 1990. pp. 1-100. In: Pathology of Domestic Animals, 4th ed. (Jubb, K.V.F. ed.), Academic Press, San Diego.

9. Rogers, W.M. and Kesten, H.D. 1963. J. Cardiovasc. Surg. 4: $175-180$. 